\title{
Greens IOT and its Green enabling Environment
}

\author{
Saijshree Srivastava, Himanshu Kumar Shukla, Surya Vikram Singh, Rudrendra Bhadur Singh \\ Department of Computer Science and Engineering, Babu Banarasi Das Institute of Technology and \\ Management, Lucknow, Uttar Pradesh, India
}

\section{Article Info}

Volume 7, Issue 4

Page Number : 104-118

\section{Publication Issue :}

July-August-2021

\section{Article History}

Accepted : 02 July 2021

Published : 08 July 2021

\section{ABSTRACT}

Internet of Thing has modified the way we work and live in the era of incredible technology development. There are various advantages of Internet of Thing that enriching our organization, it should be provoked that the Internet of Thing also consumes energy, comprises toxic pollution and E-waste. These places new hassle on the environments and smart sphere. The way of increase the profits and reduce the harm of Internet of Thing, there is an increasing craving to move toward green Internet of Thing. The future of IoT is seen in Green Internet of Thing that is environmentally friendly. To achieve that, it is very much important to put various measures to diminish carbon footprint, conserve fewer resources, and promote proficient techniques for energy usage. The main aim of green Internet of Thing, that moving headed to the machines, communications, sensors, clouds, and internet are alongside energy effectiveness and reducing carbon emission. This paper represents a thorough analysis of the contemporary continuing research work and probable technologies of green Internet of Thing with an objective to provide some clues for future green research.

Keywords : Internet of things and its overview, Application of IoT, Green IoT, Related projects, Enabling Technologies of Green IoT, Green Wireless Sensor Networks, Green Machine to Machine, Green Cloud Computing, Green Data Center Challenges and Opportunities for Green IoT, Future of Green IoT.

\section{INTRODUCTION}

The term" Internet of Things" was coined by Kevin Ashton in the year 1998 in a presentation where he mentioned that" The IoT has the ability to alter the globe, as same as the Internet did. Maybe even more so" [1].
The internet makes the sphere into a small village where things are attached to each other and with the world via global communication networks using protocols. The things include communication devices as well as physical objects, like vehicles, processor, and home appliances, which are controlled through wireless communication networks.[2] Conclusively, the potential of the IoT idea is the high effect it will

Copyright: @ the author(s), publisher and licensee Technoscience Academy. This is an open-access article distributed under the terms of the Creative Commons Attribution Non-Commercial License, which permits unrestricted non-commercial use, distribution, and reproduction in any medium, provided the original work is properly cited 
have on numerous aspects of everyday-life and activities of prospective users. From the point of view of a private user, the most obvious effects of the IoT introduction will be observable in both employed and domestic fields.

In this context, domestics, motor-assisted living, ehealth, improved learning square measure solely many samples of probable application eventualities during which the new paradigm can play a foremost role within the close to future. Similarly, from the perspective of business users, the most apparent significance will be equally observable in fields such as, automation and industrial manufacturing, logistics, business/process management, intelligent transportation of people and goods.[1]

In common language, the IoT rises to a new kind of world where nearly all the devices and appliances that we use are connected to a network. We can utilize them collectively to achieve difficult tasks that necessitate a high grade of intelligence. For this entomb association, IoT devices have embedded sensors, actuators, processors, and transceivers. IoT is a pool of several technologies that collaborate in tandem. Sensors and actuators are square measures devices, which help in work together with the physical environment. The sensors collected the data and has to be put in storage and managed wisely to get useful information from it.

Note that we tend to broadly speaking outline the term sensing element; a portable or maybe a kitchen appliance will count as a sensor as long because it provides inputs concerning its current form (internal form +atmosphere). An actuator is a machine that may use in the change in the atmosphere such as the humidity controller of an AC. [3]

\section{AN APPLICATION OF AN IOT}

Substantial changes in our environments have occurred, and some changes will occur soon because of the enlargements in IoT. However, the cost of the developments is possibly important due to the rise in e-waste, hazardous emissions, and energy usage. In this paper, the concentration will be concisely on the IoT's applications in transportation, smart city or home, personal, smart grid, habitat monitoring, health and living.[3]as addressed in fig [2].

\section{Smart Homes:}

Smart Homes are going to be as common as smart phones due to the profits offered by them such as energy saving, cutting the cost and time involved in performing actions. Some obtainable structures in a smart home include remotely controlled air conditioner, heating, lighting, i monitoring other household devices.[5] 'Smart Homes' have been an active technical research area in decades[68]. From this outlook it deals with the technological development of the living environment to offer support to inhabitants and increase their quality of life.[3]

\section{B. Smart Grid:}

Smart grid is a new trendy application of IoT. Data is automatically collected and evaluated to understand the behavior of consumers, and the pattern of usage of electricity for enlightening the efficiency of the system as well as having understanding into the economics of electricity usage.[6]

\section{Industrial IoT:}

The Industrial IoT (IIoT) is the custom of IoT in manufacturing, logistics, transportation, utilities, mining and steel, aviation and various industrial sectors. Industrial IoT is a Transformative industrializing policy that leads to enhance quality, safety, and productivity. The applications of IIoT include real time information interchange about supply among suppliers and vendors, tracking goods and programmed delivery etc. It also has great opportunity for quality control and sustainability. 


\section{Health and Living:}

The healthcare sector has benefited substantially from IoT technologies. Currently, efforts attempt to provide energy efficient solutions to help patients by supporting green hospitals and green equipment that require ultra- low or no energy sources. Performance of health care applications is improved, by embedding sensors and actuators in patients and their medicine for monitoring and tracking patients. For an illustration, by meeting and analyzing patients' body data with sensors and further delivering analyzed data to a processing center, the clinical care could monitor physiological stages of patients in realtime and make appropriate actions when required.

\section{E. Smart City:}

People tend to move to cities due to better health facilities, greater access to education and a large job market. Way of life in the city is enriched, by making it more convenient and easier for the people to get information. For illustration, according to people's needs, several interconnected organizations intelligently offer the best services (e.g., transportation, conveniences, health, etc.) to the societies. The main idea of the "smart city" has recently been presented as a strategic scheme to enable urban population development and to highlight the significance of IoT for managing it.

\section{F. Habitat Monitoring:}

The conservation of habitats is essential in sustaining provincial, national, and global ecological, commercial, and social systems. Habitat monitoring is used to recognize spatial and sequential alterations, physical transformation in the surroundings, organism changes, and changes caused by human activities or natural actions.

\section{G. Transportation:}

Recently, cars, trains, buses, bicycles, and roads have been prepared with tags, sensors, actuators, and the necessary processing power to send important information to traffic control sites. Such advanced transportation systems help to route traffic better, provide tourists with appropriate transportation information, and monitor the status of transported goods.

1) Smart Parking: The increasing availability of automobiles in current years has created a problem of finding vacant places to park the vehicles, particularly in major cities. This situation contributes to air pollution, fuel waste, and motorist frustration. This problem can be solved by introducing a Smart Parking Systems, which is an efficient and costeffective approach based on IoT technologies [59], [60].

2) Smart Traffic Congestion Detection: With the growing worldwide population, traffic problems, such as traffic congestion, etc., are increasing daily. By using the technology of vehicular ad hoc networks (VANETs), it is possible to avoid traffic congestion allowing automobiles to connect with each other and to share road information to better recognize road conditions. This will diminish carbon emissions and help to build a green environment [61], [62].

3) Smart Logistics/Shipment: Information collected through RFID, NFC, and sensors can allow real-time monitoring of the supply chain system. These technologies can also gather product-related information in real time to help enterprises to respond to changing markets in the minimum possible time. Usually, to realize customer requirement a typical enterprise requires around 120 days. In contrast, enterprises using advanced technologies (such as Wal-Mart and Metro) only need few days to accomplish customer demands [63], [64]. Furthermore, fruits, meat, and dairy foods travel thousands of miles from the production site to consumption sites and require constant monitoring to ensure quality standards. IoT enabled technologies offer great potential for improving the efficiency of the food supply chain and for helping and limit the carbon footprints. [65],[66] 


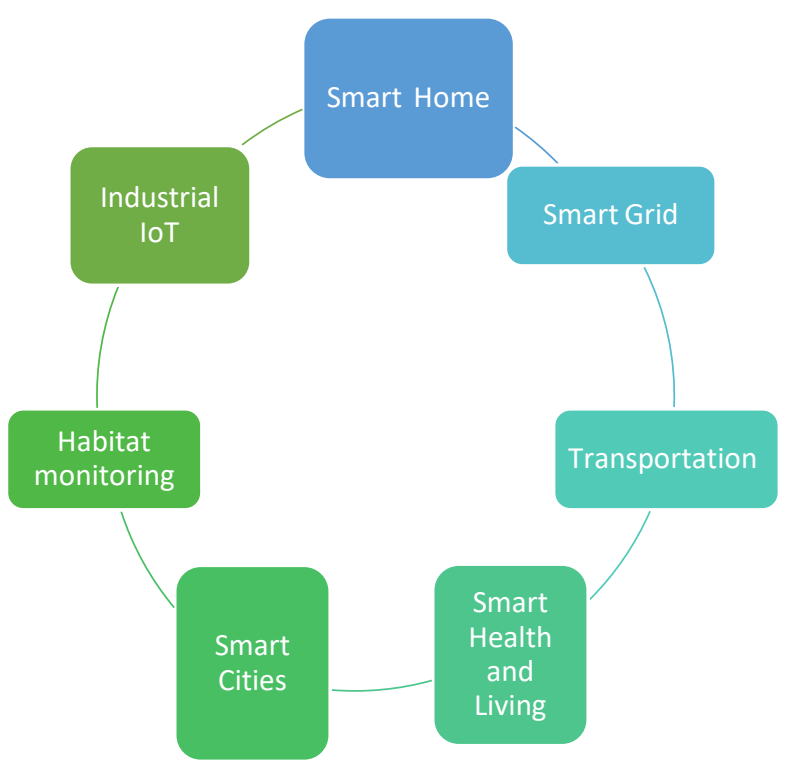

Fig 2 : An Application of an IoT

\section{Green Internet of Things Overview}

To accomplish the smart sphere development and sustainability, green Internet of Things are proposed to decrease carbon emission, and power consumption. Due to the rising in awareness of environmental issues around the world, green Internet of Things technologies concern should be taken into advantages. Greening IoT refers to the technologies that make the IoT ecological in A Pleasant way by making use of facilities and accommodation that enabling subscribers to collect, accumulate, access and manage numerous information. An indeed focus of green IoT are categorized as: green use, green design, green disposal, green manufacturing in fig [3]. The facilitative technologies for green IoT are referred to as Information and Communication Technology (ICT) technologies. Green ICT technologies refer to the facilities and storage enabling subscribers to collect, accumulate, access, and manage numerous information.

Green IoT can be described as the energy efficient measures (hardware or software) [67] implemented by IoT either to facilitate decreasing the greenhouse effect of prevailing applications and services or to decrease the influence of greenhouse effect of IoT itself. In the previous case, the benefit of IoT will aid to decrease the greenhouse effect, while in the later case further optimization of IoT greenhouse footprint will be taken care. The whole phase of green IoT should emphasis on green strategy, green manufacture, green consumption, and finally green reprocessing to have now or very small impact on the atmosphere.[7]

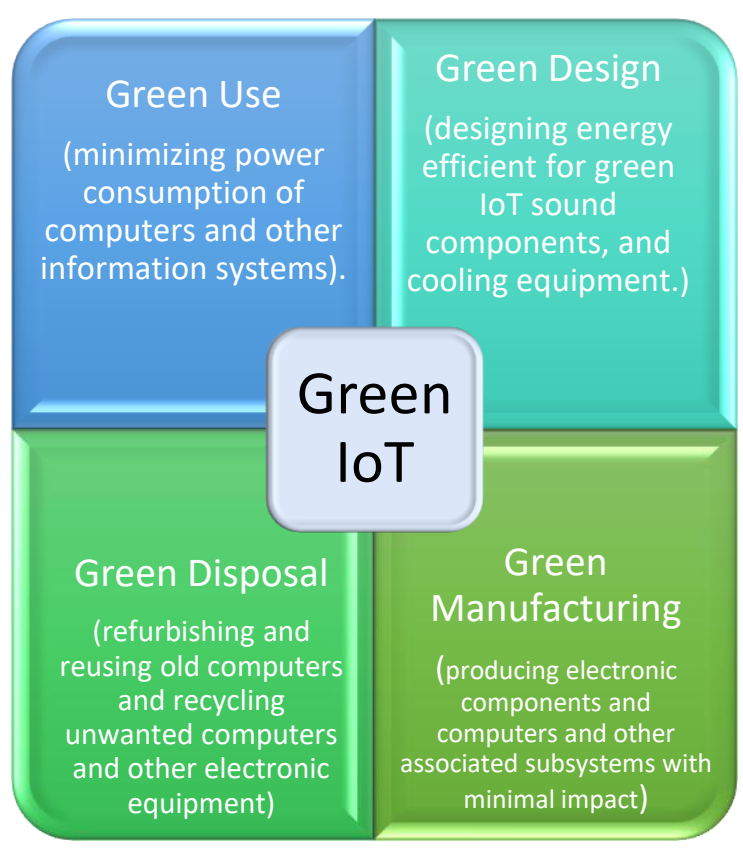

Fig 3 : An indeed focus of Green IoT

\section{Related Projects and Standarisation of Green IoT}

In recent years, a growing number of green projects have been funded to facilitate the research, experimentation, deployment, and evaluation of green techniques for IoT. Here, we review research projects and standardization efforts concerning green IoT initiatives. Governments across the world have put forth initiatives to force corporations to reduce carbon emissions, become more energy efficient, and use greener techniques. An On-going project on green IoT is concerned in fig [4]. 
$>\quad$ The EU FP7 program has supported several longterm research projects. Among them, project EARTH [28]: investigates the energy efficiency of wireless communication systems. It focuses on the theoretical and practical energy efficiency limitations of current networks to develop a new generation of energy efficient equipment, deployment strategies, and network management solutions to ensure quality of service.

$>\quad$ The EU FP7 includes another project known as Toward Real Energy-efficient Network Design (TREND) [29] focusing on energy-efficient networking. The TREND project collects power consumption data, assesses the energy-saving proficiency of technologies, protocols, architectures, and experiments with new approaches.

The EU FP7 also includes training programs to spread green network awareness, i.e., Green Net [30].

$>$ The IEEE Communication Society has also established a TSCGCC (Technical Subcommittee on Green Communications and Computing) [31]. TSCGCC works to develop and standardize energy-efficient communications and computing. It also provides opportunities to interact and exchange technical ideas, to identify $\mathrm{R} \& \mathrm{D}$ challenges, and to collaborate on solutions for the development of energy-sustainable, supply-saving, and environmentally friendly green networks and computing tools.

$>$ Another consortium, Green Touch [32], consists of approximately 30 leading ICT companies and research institutes that have come together to transform communications and data networks. Green Touch places emphasis on reducing the CO2 footprint of ICT devices and networks.

$>$ Cool silicon [33] focuses on building the technological basis necessary to support a massive increase in energy efficiency in the ICT sector.

$>$ Cool silicon marks high energy efficiency for micro and nanotechnologies and green communication systems and seeks to develop energy-efficient sensors.

TABLE 1. Project activities for Green IoT

\begin{tabular}{|l|l|l|}
\hline Projects & Status & Green Perspective \\
\hline M2M & Continuing & $\begin{array}{l}\text { M2M } \\
\text { standardization. }\end{array}$ \\
\hline TREND & Continuing & $\begin{array}{l}\text { Energy efficient } \\
\text { networking. }\end{array}$ \\
\hline $\begin{array}{l}\text { Green } \\
\text { Net }\end{array}$ & Continuing & $\begin{array}{l}\text { Training of green } \\
\text { communication. }\end{array}$ \\
\hline TSCGCC & Advancement & $\begin{array}{l}\text { Energy efficient } \\
\text { computing } \\
\text { standardization. }\end{array}$ \\
\hline $\begin{array}{l}\text { Green } \\
\text { Touch }\end{array}$ & Advancement & $\begin{array}{l}\text { Reduction of co2 } \\
\text { footprint of ICT. }\end{array}$ \\
\hline $\begin{array}{l}\text { Cool } \\
\text { Silicon }\end{array}$ & Continuing & $\begin{array}{l}\text { Energy efficient in } \\
\text { the ICT. }\end{array}$ \\
\hline $\begin{array}{l}\text { GREEN } \\
\text { T }\end{array}$ & Continuing & $\begin{array}{l}\text { Energy efficiency in } \\
\text { heterogenous wireless } \\
\text { networks. }\end{array}$ \\
\hline $\begin{array}{l}\text { GO } \\
\text { Energi }\end{array}$ & Continuing & $\begin{array}{l}\text { Energy efficiency in } \\
\text { household, trade } \\
\text { sector. }\end{array}$ \\
\hline $\begin{array}{l}\text { EPC } \\
\text { Global } \\
\text { Green } \\
\text { grid }\end{array}$ & Advancement & $\begin{array}{l}\text { RFID Technology. } \\
\text { Entinuing } \\
\text { Eata center. }\end{array}$ \\
\hline
\end{tabular}

The green Grid [38], a global consortium, specifically targets the energy efficiency of the data centres. Full deployment of IoT relies on several on-going contributions from various projects and companies.

$>$ RFID and the Internet Engineering Task Force (IETF) is working on two major initiatives to 
make IPV6 suitable for low end devices: 6LoWPAN [39] and Routing Over Low power and Lossy networks (ROLL) [40].

$>$ Celtic-Plus [34] is an industry-driven EU research initiative that supports various projects.

$>$ Among them, the GREEN-T [35] mainly focus on new approaches to optimize energy efficiency in heterogeneous wireless networks.

$>$ Similarly, the green IT [36], an initiative of the Japan, the energy efficiency techniques of data centres, systems and demonstrations have been targeted.

$>$ The Danish Energy Saving Trust (Go' Energi) [37] upholds energy efficiency in households, the public sector, and the commercial and industrial sectors for all types of energy use.

$>$ In the United Kingdom, Mobile VCE targets the parallel evolution of green architectures and techniques in the Green Radio project [38]. EPC global [41], the European Telecommunications Standards Institute (ETSI) [42], European Committee for Standardization (CEN) [43], and the International Standard Organization (ISO) [44] are making major contributions to standardize RFID technology. ETSI has also bring out the M2M Technical Committee [45] to standardize activities related to IoT for M2M and WSN.

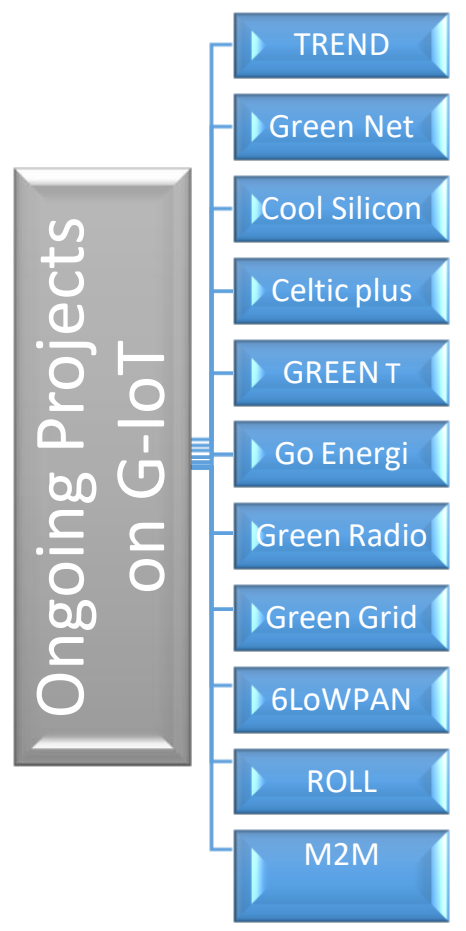

Fig 4 : An on-going project on green IoT

\section{Enabling Technology of Green Internet of Things}

Greening ICT is enabling tools for green IoT which comprises green RFID (Radio-Frequency Identification), green WSN (Wireless Sensor Networks), green M2M (Machine to Machine), green CC (Cloud Computing), green DC (Data Center). Therefore, greening ICT technologies perform a vital role to green Internet of Things and provide many profits to society such as reducing the energy that is used for constructing, producing and delivering ICT devices and tools as discuss in table [2].

\section{Green RFID TAGS:}

RFID is the combined term of RF and ID where RF denotes to wireless communication technology, and ID implies as tag identification information. It is measured as the promising wireless communication system used to enable IoT.[8]

RFID have several RFID tags and a very small subset of tag readers. The main objective of RFID tags is storing information regarding the objects to which they are attached. The simple process is the information flow is triggered by RFID tag readers 
through transmitting a query signal, followed with the responses of nearby RFID tags. Mostly, the transmission range of RFID systems is very low (i.e., a few meters). Besides, various bands (i.e., from low frequencies at $124-135 \mathrm{kHz}$ up to extreme- high frequencies at $860-960 \mathrm{MHz}$ ) are used to perform transmission.

There area unit 2 varieties of RFID tags: active and passive. The passive tags don't have on board batteries, and produce energy from the reader signal using the principle of induction while Active tags have batteries operating the signal programmed and intensifying the transmission ranges, [9] RFID plays a major role in applications that help to promote a greener world by decreasing vehicle emissions, monitoring the health of wildlife, conserving energy use in buildings, improving waste disposal, etc.

\section{Green Wireless Sensor Network:}

The amalgamation of wireless communication and sensing have directed to the wireless sensor networks. WSNs denote the critical technology which has made IoT succeed. A sensor is a grouping of a massive number of small, low-power and low-cost electronic devices. Numerous sensors and base station (BS) nodes signify the components of WSN. Each sensor node contains sensing, power, processing and communication unit which was discussed in [10]. Sensor nodes are being structured around the sphere, computing local and global environmental circumstances such as weather, pollution, and farming fields, and so on. Each sensor node examines from atmospheres such as temperature, pressure, sound, accelaration, humidity, etc. WSNs have numerous uses such as fire detection [11-12], object tracking [13-15], environmental monitoring [10, 1618], progressing constraints in the military [19], monitor machine health monitoring, industrial process monitoring[10].Various application of WSN are concerning in fig [5].

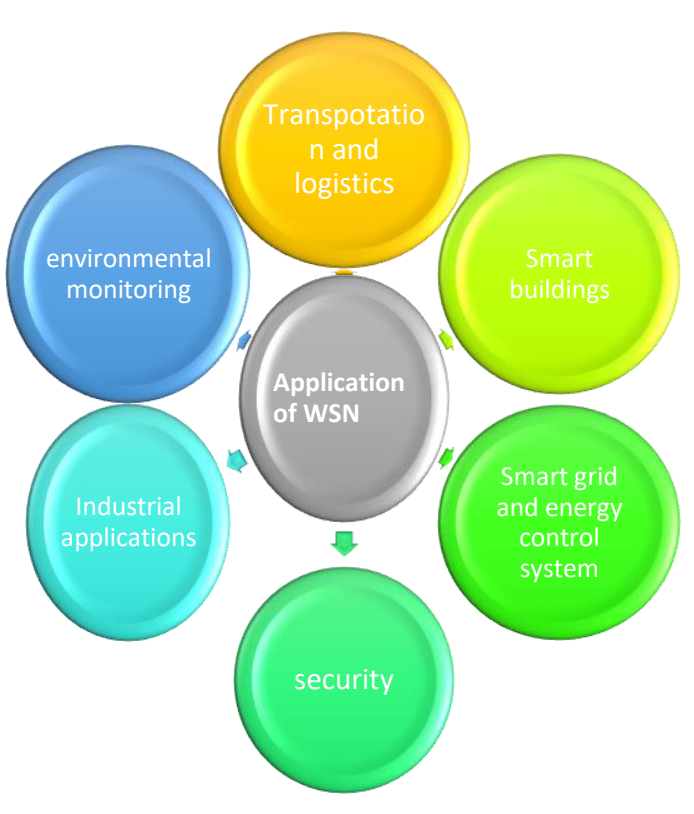

Fig 5 : An application of Wireless Sensor Network

Regarding green WSN, the subsequent techniques should be embraced [20] [21][22][23]:

1. Make sensor nodes only work, when necessary, while spending the rest of their lifetime in a sleep mode to save energy consumption;

2. Energy depletion (e.g., wireless)

3. Charging, utilizing energy harvesting mechanisms which generate power from the environment (e.g, sun, kinetic energy, vibration, temperature differentials, etc.);

4. Radio optimization techniques (e.g., transmission power control, modulation optimization, cooperative transmission, reversing antennas, Energy-Efficient cognitive radio (CR));

5. Data reduction tool (e.g., Adaptive sampling, aggregation, network coding, compression);

6. Energy-Efficient routing methods (e.g., Energy as per routing metric, cluster manners, Multipath routing, node mobility, relay node placement.

\section{Green Machine to Machine:}

Regarding M2M communications, massive M2M nodes which wisely collect the monitored data is 
deployed in M2M domain. In network domain, the wired/wireless network conveys the collected data to the base station. The BS further supports several M2M applications over network in the application domain. Green M2M and the immense machines comprise in M2M communications. They will use several energies, mainly in the M2M field. The enabling technologies issue of M2M communication for the HEMS (Home Energy Management System) in the smart grid [2] discussed by Niyato et al. [24]. Following techniques might be used to rise energy efficiency for greening IoT:

1) Intelligently adjust power transmission;

2) Efficient communication protocols essential for distributing the computing techniques;

3) Activity scheduling of node used to shift several nodes to sleeping mode while observance the functionality of the original network;

4) Mechanisms for Energy savings;

5) Employ energy harvesting and the benefits of CR.

$\mathrm{CR}$ is a fusion of electronic network and a computer network. It is used in developing an intelligent M2M communication among CR-based "smart meters" to RAPM (Remote-Area Power Management). The reason behind the fusion is to maximize the power efficiency of electricity distribution and the spectrum efficiency. Moreover, the united network design based on the flexible, high-capacity and cost-effective 4G (LTE) technology was discussed by Vo et al [], which helps M2M connectivity in an end on fashion.

\section{Green Cloud Computing}

In cloud computing, resources are considered as services, i.e., IaaS (infrastructure as a service), PaaS (platform as a service) and SaaS (software as a service). Based on users' demands, cloud computing elastically offers several resources (e.g., high-capacity storage space, high-performance computing resources) to users.[2] The key aim of green cloud computing is to support the utilization of sustainable products which are simply recycled and reused. Hence, an idea of green computing with a focus on information technologies are given by shuja et al. [2]

The primary goal of Green Cloud Computing is to ease the use of precarious materials, maximize energy consumption, and enhance the recyclability of old products and wastes. Moreover, it can be achieved by product durability resource allocation, and paperless virtualization or appropriate power management. The basic architecture is addressed in fig [6].

With respect to green CC, potential solutions are presented as follows [8], [25] [26]:

1) Implementation of hardware and software that reduce energy consumption. In this regard, hardware solutions should aim at designing and manufacturing devices which consume a reduced amount of energy. Software solutions should try to offer efficient software designs consuming less energy with least resource utilization;

2) Power-saving virtual machine (VM) techniques (e.g., VM consolidation, VM migration, VM placement, VM allocation);

3) Various energy-efficient resource allocation mechanisms (e.g., gossip-based resource allocation, auction-built resource allocation) and associated task scheduling tools;

4) Effective and accurate models and evaluation approaches regarding energy-saving policies;

5) Green CC schemes based on cloud supporting technologies (e.g., networks, communications, etc.). 


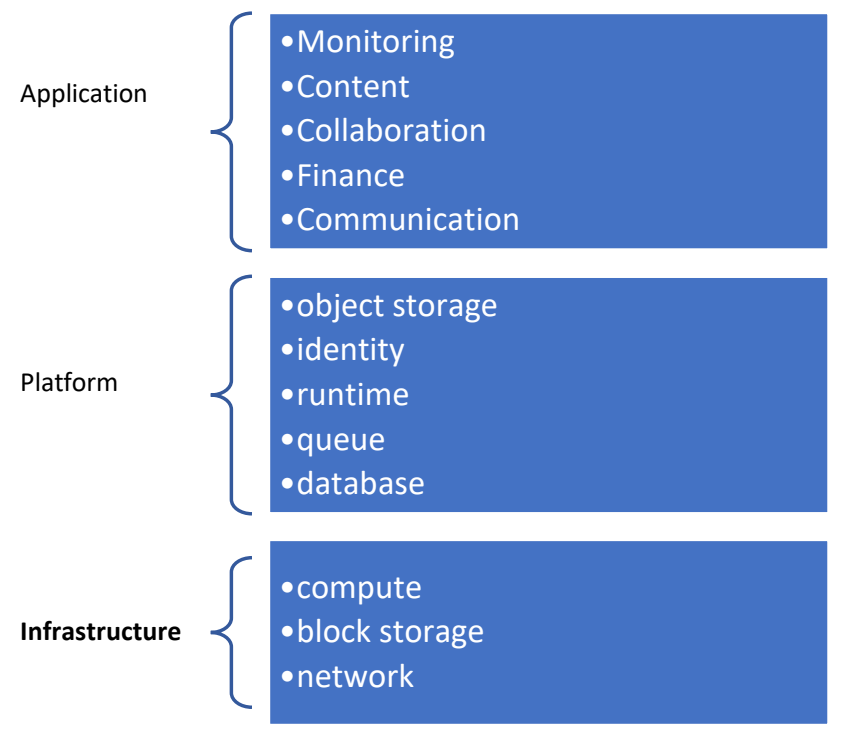

Fig 6 : Basic architecture of cloud computing

\section{Green Data Center:}

Green Data Center is a new technology and an origin for data storage, data organization, and data distribution. These data are made by users, systems, things, etc. Dealing with different data and applications, data center utilizes huge extents of energy with huge operational costs and important $\mathrm{CO} 2$ Footprints. Moreover, generation of big data is rising by various ubiquitous things such as mobile devices, sensors, etc. On the way of the smart world, the energy efficiency for data center becomes more pressing [9]. Moreover, green data center is providing data services for cloud-abetted (MANET) in 5G [27]. Advanced technologies are used for low production construction materials, reducing the house paints and carpets, low emission construction tools, ecological shaping, using alternative energy (i.e., evaporative cooling heat pumps, photovoltaic and heat pumps).

About green data center, probable techniques to improve energy efficiency can be accomplished from the following aspects:

1) Use renewable or inexperienced sources of energy (e.g., wind, water, solar energy, heat pumps etc.);

2) Consume efficient dynamic powermanagement tools (e.g., vSphere and Turbo boost);
3) Construct additional energy-efficient hardware (e.g., Make use of the benefits of (vary-on/vary off) VOVO methods and (dynamic voltage and frequency scaling) DVFS methods;

4) Construct novel energyefficient knowledge centre architectures (e.g., nano data centres) to achieve power conservation;

5) Construct energy-aware routing algorithms to consolidate traffic flows to a set of the network and power off the idle devices;

6) Construct effective and correct knowledge centre power models;

7) Draw support from communication and computing techniques (e.g., optical communication, $\mathrm{VM}$ migration, placement optimisation, etc

TABLE 2. Green ICT enabling technologies:

\begin{tabular}{|l|l|}
\hline SCHEMES & TECHNIQUES \\
1)Green & $\begin{array}{l}\text { 1]Reduce the sizes of RFID tags to } \\
\text { decrease the extent of non- } \\
\text { degradable raw material used in } \\
\text { their manufacturing; } \\
\text { 2]Energy efficient algorithms and } \\
\text { protocol for tag estimation, } \\
\text { altering transmission power level } \\
\text { dynamically etc. }\end{array}$ \\
\hline 2)Green & $\begin{array}{l}\text { 1] Energy efficient techniques } \\
\text { (e.g., cluster constructions, energy } \\
\text { as a routing metric, multipath } \\
\text { steering etc.). } \\
\text { 2] Energy depletion (e.g.; wireless } \\
\text { charging, energy harvesting } \\
\text { mechanism etc.). } \\
\text { 3]Radio optimization technique } \\
\text { (e.g.; transmission power control, } \\
\text { modulation optimization, energy } \\
\text { efficient CR). }\end{array}$ \\
\hline
\end{tabular}




\begin{tabular}{|l|l|}
\hline 3) Green & $\begin{array}{l}\text { 1] Wisely adjust the transmission } \\
\text { power (e.g.; to the nominal energy } \\
\text { level). } \\
\text { 2] Joint energy saving mechanisms } \\
\text { (e.g., with overload protection and } \\
\text { resource allocation). }\end{array}$ \\
\hline 4)Green CC & $\begin{array}{l}\text { 1] Green CC schemes based on } \\
\text { cloud supporting techniques } \\
\text { (network, communications etc.); } \\
\text { 2] Power saving virtual machine } \\
\text { techniques; } \\
\text { 3] Effective and evaluative } \\
\text { approaches regarding power } \\
\text { saving policies. }\end{array}$ \\
$\begin{array}{l}\text { 1] Use renewable or green sources } \\
\text { of energy (e.g.; solar energy, heat } \\
\text { pumps etc.). } \\
\text { 2] Design effective and accurate } \\
\text { data center power models. } \\
\text { 3] Draw support from } \\
\text { communication and computing } \\
\text { methods }\end{array}$ \\
\hline
\end{tabular}

\section{Challenges and opportunities of green IoT}

Green technologies have a significant role in enabling the energy-efficient IoT. There are various challenging issues that should be addressed in fig [7]. Here, we summarize them and focus the crucial issues that need further deliberation.

\section{Green IoT Architectures:}

For IoT, a standard architecture, such as the ISO OSI model or the TCP/IP model, is needed to enable communication across various applications and heterogeneous networks that have a broad range of devices. Furthermore, it is essential to understand how to integrate energy efficiency across the whole architecture. Communication should be energy proficient in both the devices and the protocols. Likewise, the applications should be energy efficient to make sure that their overall impact on the surroundings is least. As is evident from Section V, academia and the industry area must work together to support and normalize the green IoT paradigm making the investigation of green IoT architectures a priority.

\section{Green Infrastructure}

Subjecting energy efficient infrastructure for IoT can be accomplished through a clean-slate restructure method. Though, due to the complexity of deploying a radically new infrastructure (or even adapting an existing infrastructure over time), this area of research is less focused and requires further attention. Accordingly, many interesting issues such as quantifying the potential benefits of designing new energy-efficient infrastructure while efficiently exploiting the current infrastructure, remain open at both the architectural and operational levels.

\section{Green Spectrum Management}

At present, handlers are confined to the limited RF spectrum, which is quite congested and difficult to use optimally. The cognitive radio approach [47] allows devices to sense the various RF channels and to tune both transmission and reception dynamically to avoid interference with concurrent users. The cognitive radio approach brings many benefits to green mobile services [48]. Although the cognitive radio efficiently manages the spectrum, it relies on continuous monitoring of the RF spectrum, which may cause it to consume more energy. In order to explore the full potential of cognitive radios more analysis in this area is needed to identify trade offs between efficient dynamic spectrum management and efficient spectrum sensing. Current efforts are limited to simulation studies, thereby providing an opportunity to develop and experiment with the cognitive radio hardware.

\section{Green Communication}

The energy-efficient communication faces many challenges such as providing a continuous energy supply to objects in loop and supporting energy- 
efficient communication protocols that enable peers to communicate in a reliable manner, etc. Furthermore, cyber physical systems (CPS) [49] are evolving due to the use of sensors, M2M, and energy harvesting mechanisms to monitor and control physical environments. Since CPS and M2M directly interfere with the physical world, the balance among performance, safety, and energy efficiency of CPSs and M2M should be a high priority for investigators and developers. Future research that focuses on integrating M2M for ubiquitous services will be critical for developments in this area. Alongside energy-efficient mechanisms for IoT, several attempts are underway to discover new energy sources that can provide a new height to green IoT [50]. Efficient adoption of new energy sources, such as wind, solar, thermal, and vibration to assist the current green IoT appear promising. Furthermore, scalability will be a major concern for green IoT applications. Tethering and multi hopping can improve the scalability [51][52]. Tethering enables groups of users to communicate directly with a host in an ad hoc manner, whereas the host is connected to the Internet. Multi hopping is a common technique to save energy and overcome the scalability issue [53][55]. Another potential solution to address the scalability problem involves the efficient exploitation of the mobility of users and objects in the network [56][57]. This opens an opportunity to find a commutation between energy efficiency and using mobility to mark the scalability problem.

\section{E. Green Security and QoS}

Provisioning Security and privacy are major concerns for IoT deployment. Implementing of security algorithms requires a substantial amount of processing from devices. The potential of energy efficient, secure mechanisms, which are still in their infancy, should encourage more research and development in this area [58]. Since IoT involves both resource-constrained devices, such as RFID and sensor nodes, and high-end data servers, it is important to find and exploit trade-offs to provide security among heterogeneous devices in the green IoT paradigm. Generally, security is often viewed as an add-on to the system. But, in the case of green IoT, security must be given high priority and should be considered early during the design phase. Along with the security, we need to investigate appropriate mechanisms that consider both energy consumption and the required QoS.

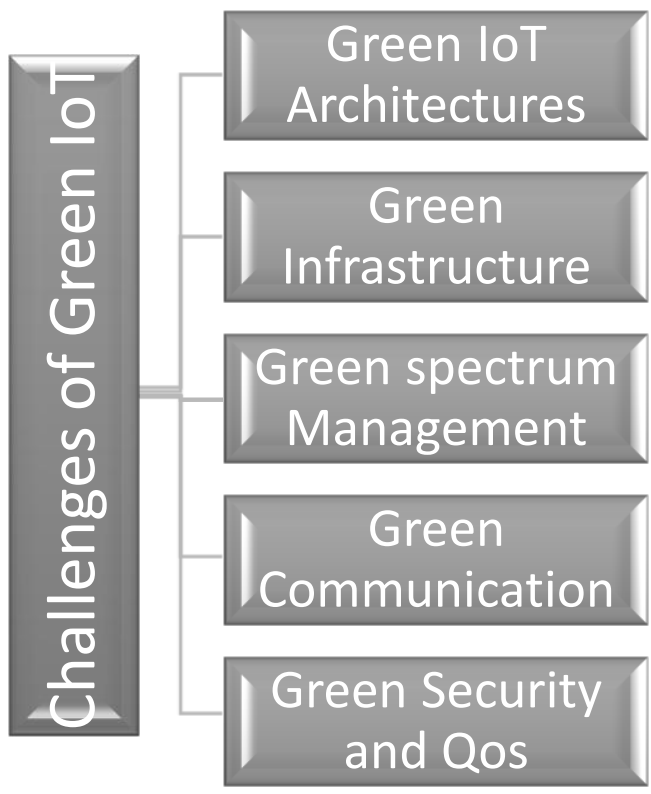

Fig 7 : Challenges of Green IoT.

\section{Future of Green IOT}

The future of green IoT will change our tomorrow atmosphere to become healthier and green, very high QoS, socially and globally sustainable and economically also. The following future research directions and open challenges and issues concerning green IoT, are seen:

1) There is a necessity for UAV to replace a huge number of IoT devices especially, in agriculture, traffic and monitoring, which will help to moderate power consumption and pollution. 
2) To reduce the use of energy, M2M communication acts an important role, hazardous emissions.

3) The radio frequency energy harvest should be taken into deliberation, in order to accomplish energy-balancing for supporting green communication between IoT devices.

4) Further research is needed to improve the design of IoT devices which helps to reduce $\mathrm{CO} 2$ emission and the energy usage. For green environmental life the most critical task for smart is saving energy and reducing the $\mathrm{CO} 2$ emission.

\section{CONCLUSION}

The technology development in the $21^{\text {st }}$ era has many advantages. In this paper we study and find out most critical technologies of green IoT and keeping our environment green and smarter. Specifically, the summary regarding IoT and green IoT has been performed. Related projects have been also be standardized in this survey and continuing projects are described. The technologies related to green IoT including five green ICT (i.e., green RFID (RadioFrequency Identification), green WSN, green M2M, green CC (Cloud Computing), green DC (Data Center)) have been introduced. Challenges and opportunities play a major role in development of GIoT. Future of green IoT have been marked upon for efficiently and effectively enlightening the green IoT based applications. This research is based on anyone who wishes to know about green IoT and its future trends are effectively and essentially understand the enabling technologies if green IoT and its future views.

\section{REFERENCES}

[1]. Mahmoud A. M. Albreem*, Ayman A. ElSaleh*, Muzamir Isa†, Wael Salah‡, M. Jusoh§,
M.M Azizan† and A Ali†: Green Internet of Things (IoT): An Overview, · Nov., 2017.

[2]. S. H. Alsamhi, Ou Ma, M. Samar Ansari, Qingliang Meng: Greening Internet of Things for Smart Everythings with A Green Environment Life: A Survey and Future Prospects.

[3]. L. Atzori, A. Iera, G. Morabito, The internet of things: A survey, Computer networks, 54 (2010) 2787-2805

[4]. Pallavi Sethi and Smruti R.Sarangi: Internet of Things: Architectures, Protocols, and Applications, Jan., 2017.

[5]. Surapon Kraijak, Panwit Tuwanut: A SURVEY ON IOT ARCHITECTURES, PROTOCOLS, APPLICATIONS, SECURITY, PRIVACY, REAL-WORLD IMPLEMENTATION AND FUTURE TRENDS.

[6]. Kavleen Kour, Jaspreet Kour, Parminder Singh: Smart Applications of Internet of Things, 2018 First International Conference on Secure Cyber Computing and Communication (ICSCCC).

[7]. P Lynggaard, K E Skouby: Smart Home and Smart City Solutions enabled by 5G, IoT, AAI and CoT Services, 2014 IEEE.

[8]. Faisal Karim Shaikh, Member, IEEE, Sherali Zeadally, Senior Member, IEEE: Enabling Technologies for Green Internet of Things, 2015.

[9]. C. Zhu, V.C. Leung, L. Shu, E.C.-H. Ngai, Green Internet of Things for the smart world, IEEE Access, 3 (2015) 2151-2162

[10]. B. Prabhu, N. Balakumar, A.J. Antony, Wireless Sensor Network Based Smart Environment Applications, (2017).

[11]. J. Lloret, M. Garcia, D. Bri, S. Sendra, A wireless sensor network deployment for rural and forest fire detection and verification, sensors, 9 (2009) 8722-8747.

[12]. Y.E. Aslan, I. Korpeoglu, Ö. Ulusoy, A framework for use of wireless sensor networks 
Saijshree Srivastava et al Int. J. Sci. Res. Comput. Sci. Eng. Inf. Technol, July-August-2021, 7 (4) : 104-118

in forest fire detection and monitoring, Computers, Environment and Urban Systems, 36 (2012) 614-625.

[13]. F. Viani, L. Lizzi, P. Rocca, M. Benedetti, M. Donelli, A. Massa, Object tracking through RSSI measurements in wireless sensor networks, Electronics Letters, 44 (2008) 653654.

[14]. G. Han, J. Shen, L. Liu, A. Qian, L. Shu, TGMCOT: energy efficient continuous object tracking scheme with two-layer grid model in wireless sensor networks, Personal and Ubiquitous Computing, 20 (2016) 349-359.

[15]. G. Han, J. Shen, L. Liu, L. Shu, BRTCO: a novel boundary recognition and tracking algorithm for continuous objects in wireless sensor networks, IEEE Systems Journal, (2017).

[16]. F. Wu, C. Rüdiger, M.R. Yuce, Real-Time Performance of a Self-Powered Environmental IoT Sensor Network System, Sensors, 17 (2017) 282.

[17]. C.A. Trasviña-Moreno, R. Blasco, Á. Marco, R. Casas, A. Trasviña-Castro, unmanned aerial vehicle based wireless sensor network for marine-coastal environment monitoring, Sensors, 17 (2017) 460.

[18]. D. Sharma, Low-Cost Experimental set up for Real Time Temperature, Humidity Monitoring through WSN, International Journal of Engineering Science, 4340 (2017).

[19]. B. Prabhu, N. Balakumar, A.J. Antony, Evolving Constraints in Military Applications Using Wireless Sensor Networks, (2017).

[20]. F. K. Shaikh, S. Zeadally, and E. Exposito, "Enabling technologies for green Internet of Things," IEEE Syst. J., to be published.

[21]. Y.-H. Lin, Z.-T. Chou, C.-W. Yu, and R.-H. Jan, "Optimal and maximized configurable power saving protocols for corona-based wireless sensor networks," IEEE Trans. Mobile Comput., to be published.
[22]. G. Anastasi, M. Conti, M. Di Francesco, and A. Passarella, "Energy conservation in wireless sensor networks: A survey,"AdHocNetw.vol.7, no. 3, pp. 537-568, May 2009.

[23]. T. Rault, A. Bouabdallah, and Y. Challal, "Energy efficiency in wireless sensor networks: A top-down survey," Comput. Netw., vol. 67, pp. 104-122, Jul. 2014.

[24]. D. Niyato, L. Xiao, P. Wang, Machine-tomachine communications for home energy management system in smart grid, IEEE Communications Magazine, 49 (2011).

[25]. F. Farahnakian et al., "Using ant colony system to consolidate VMs for green cloud computing," IEEE Trans. Services Comput., vol. 8, no. 2, pp. 187-198, Mar./Apr. 2015.

[26]. Y.-J. Chiang, Y.-C. Ouyang, and C.-H. Hsu, "An efficient green control algorithm in cloud computing for cost optimization," IEEE Trans. Cloud Comput., vol. 3, no. 2, pp. 145-155, Apr./Jun. 2015.

[27]. N.D. Han, Y. Chung, M. Jo, Green data centers for cloud assisted mobile ad hoc networks in 5G, IEEE Network, 29 (2015) 7076.

[28]. EARTH, Accessed Feb. 20, 2014. [Online] Available: https://www.ictearth.eu/

[29]. TREND, Accessed Feb. 20, 2014. [Online]. Available: www.fp7-trend. eu/

[30]. Green Net, Accessed Jan.2, 2014. [Online]. Available: www.fp7-greenet. Eu

[31]. Green Comm. \& Comp. [Online]. Available: www.comsoc.org/about/ committees/emerging \#gcc

[32]. Green Touch, Accessed Feb. 20, 2014. [Online]. Available: www. greentouch.org/

[33]. Cool Silicon, Accessed Oct. 1, 2014. [Online]. Available: www.coolsilicon.de/

[34]. Celtic Plus, Accessed Dec.1, 2014. [Online]. Available: www.celticplus.eu 
[35]. GREEN-T greent.av.it.pt Accessed Feb. 28, 2014.

[36]. Green IT Initiatives in Japan, Accessed Feb. 28, 2014. [Online]. Available www.meti.go.jp/english/policy/

[37]. Go' Energi, Accessed Dec. 12, 2012. [Online]. Available: www. savingtrust.dk

[38]. Green Radio, Accessed Feb. 2, 2013. [Online]. Available: www.mobilevce.com/green-radio

[39]. The Green Grid, Accessed Dec. 28, 2012. [Online]. Available: www.thegreengrid.org

[40]. N. Kushalnagar et al., "6LoWPAN," Internet Eng. Task Force, Praque, Czech Republic, RFC 4919, 2007.

[41]. ROLL, Accessed Mar. 4, 2014. [Online]. Available: https://datatracker. ietf.org/wg/roll/

[42]. EPC global, Accessed Feb. 2, 2012. [Online]. Available: www.gs1.org/ epc global

[43]. ETSI, Accessed Feb. 2, 2014. [Online]. Available: www.etsi.org [112] CEN, Accessed Feb. 1, 2014. [Online]. Available: www.cen.eu

[44]. ISO, Accessed Jan. 18, 2014. [Online]. Available: www.iso.org [114] ETSI M2M Standardization, Accessed Mar. 4, 2014. [Online]. Available: www.etsi.org/technologiesclusters/technologies/m2m.

[45]. ECMA-340, NFCIP-1, Accessed Mar. 23, 2014. [Online]. Available: www.ecmainternational.org/publications/standards/ecma340.htm

[46]. The ZigBee alliance, Accessed Mar. 2, 2014. [Online]. Available: www. zigbee.org

[47]. I. F. Akyildiz, W.-Y. Lee, M. C. Vuran, and S. Mohanty, "A survey on spectrum management in cognitive radio networks, "IEEE Commun. Mag., vol. 46, no. 4, pp. 40-48, 2009.

[48]. D. Grace, J. Chen, T. Jiang, and P. D. Mitchell, 'Using cognitive radio to deliver 'green' communications," in Proc. CROWNCOM, Apr. 2009, pp. 1-6.
[49]. E. A. Lee, "Cyber physical systems: Design challenges," in Proc. ISORC, 2008, pp. 363369.

[50]. "Mobile networks go green-minimizing power consumption and leveraging renewable energy," ABI Res., New York, NY, USA, Tech. Rep, 2008.

[51]. A. Sharma, V. Navda, R. Ramjee, V. Padmanabhan, and E. Belding, "Cool-tether: Energy efficient on-the-fly wifi hot-spots using mobile phones," in Proc. ENET, 2010, pp. 109120.

[52]. H. Lei, X. Wang, and P. H. J. Chong, "Opportunistic relay selection in future green multi hop cellular networks," in Proc. IEEE 72nd VTC, 2010, pp. 1-5.

[53]. I. F. Akyildiz, W. Su, Y. Sankara subramaniam, and E. Cayirci, "Wireless sensor networks: A survey," Comput. Netw., vol. 38, no. 4, pp. 393-422, Mar. 2002.

[54]. J.N.Al-Karaki and A.E.Kamal,"Routing techniques in wireless sensor networks: A survey," IEEE Wireless Commun., vol. 11, no. 6, pp. 6-28, Dec. 2004.

[55]. N. A. Pantazis and D. D. Vergados, "A survey on power control issues in wireless sensor networks," IEEE Commun. Surveys Tuts., vol. 9, no. 4, pp. 86-107, Fourth Quarter 2007.

[56]. Y. Wang, H. Dang, and H. Wu, "A survey on analytic studies of delay to learnt mobile sensor networks,"WCMC, vol.7no.10, pp. 1197-1208, Dec. 2007.

[57]. M. Di Francesco, M. Das, and G. Anastasi, "Data collection in WSNs with mobile elements: A survey," Trans. Sens. Netw., vol. 8, no. 1, pp. 1-31, 2011.

[58]. L. Caviglione, A. Merlo, and M. Migliardi, "What is green security?" in Conf. Rec. IEEE 7th IAS Annu. Meeting, 2011, pp. 366-37.

[59]. M. Y. I. Idris, Y. Y. Leng, E. M. Tamil, N. N. Noor, and Z. Razak, "Car park system: A 
Saijshree Srivastava et al Int. J. Sci. Res. Comput. Sci. Eng. Inf. Technol, July-August-2021, 7 (4) : 104-118

review of smart parking system and its technology," Inf. Technol. J., vol. 8, no. 2, pp. 101-113, 2009.

[60]. S. Yoo et al., "PGS: Parking guidance system based on WSN," in Proc. ISWPC, 2008, pp. 218-222.

[61]. Y.Xuetal., "Data collection for the detection of urban traffic congestion by vanets ," in Proc. APSCC, 2010, pp. 405-410.

[62]. Y. Xu, Y. Wu, J. Xu, and L. Sun, "Efficient detection scheme for urban traffic congestion using buses," in Proc. 26th Int. Conf. WAINA, 2012, pp. 287-293.

[63]. R. Yuan et al., Value chain-oriented RFID system framework and enterprise application, 2007.

[64]. METRO Group Future Store Initiative. [Online]. Available: www. futurestore.org

[65]. A. Ilic, T. Staake, and E. Fleisch, "Using sensor information to reduce the carbon footprint of perishable goods," IEEE Pervasive Comput., vol. 8, no. 1, pp. 22-29, Jan.-Mar. 2009.

[66]. A. Dada and F. Thiesse, "Sensor applications in the supply chain: The example of quality-based issuing of perishables," Internet Things, vol. 4952, pp. 140-154, 2008.

[67]. S. Srivastava and S. Prakash, "An Analysis of Various IoT Security Techniques: A Review," 2020 8th International Conference on Reliability, Infocom Technologies and Optimization (Trends and Future Directions) (ICRITO), 2020, pp. 355-362, doi: 10.1109/ICRITO48877.2020.9198027

[68]. Srivastava S., Prakash S. (2020) Security Enhancement of IoT Based Smart Home Using Hybrid Technique. In: Bhattacharjee A., Borgohain S., Soni B., Verma G., Gao XZ. (eds) Machine Learning, Image Processing, Network Security and Data Sciences. MIND 2020. Communications in Computer and Information
Science, vol 1241. Springer, Singapore. https://doi.org/10.1007/978-981-15-6318-8_44

\section{Cite this article as :}

Saijshree Srivastava, Himanshu Kumar Shukla, Surya Vikram Singh, Rudrendra Bhadur Singh, "Greens IOT and its Green enabling Environment ", International Journal of Scientific Research in Computer Science, Engineering and Information Technology (IJSRCSEIT), ISSN : 2456-3307, Volume 7 Issue 4, pp. 104-118, July-August 2021. Available at doi : https://doi.org/10.32628/CSEIT217424 Journal URL : https://ijsrcseit.com/CSEIT217424 | 